\title{
Indoor Positioning of Shoppers Using a Network of Bluetooth Low Energy Beacons
}

\author{
Patrick Dickinson, Gregorz Cielniak, Olivier Szymanezyk \\ University of Lincoln \\ Lincoln, UK \\ pdickinson, gcielniak, oszymanezyk@lincoln.ac.uk
}

\author{
Mike Mannion \\ RNF Digital Innovation Ltd. \\ Leamington Spa, UK \\ mike.mannion@rnfdigital.com
}

\begin{abstract}
In this paper we present our work on the indoor positioning of users (shoppers), using a network of Bluetooth Low Energy (BLE) beacons deployed in a large wholesale shopping store. Our objective is to accurately determine which product sections a user is adjacent to while traversing the store, using RSSI readings from multiple beacons, measured asynchronously on a standard commercial mobile device. We further wish to leverage the store layout (which imposes natural constraints on the movement of users) and the physical configuration of the beacon network, to produce a robust and efficient solution. We start by describing our application context and hardware configuration, and proceed to introduce our nodegraph model of user location. We then describe our experimental work which begins with an investigation of signal characteristics along and across aisles. We propose three methods of localization, using a "nearest-beacon" approach as a base-line; exponentially averaged weighted range estimates; and a particlefilter method based on the RSSI attenuation model and Gaussian-noise. Our results demonstrate that the particle filter method significantly out-performs the others. Scalability also makes this method ideal for applications run on mobile devices with more limited computational capabilities.
\end{abstract}

Keywords-indoor positioning; beacons; Bluetooth; shoppers; localization; BLE; particle filter.

\section{INTRODUCTION}

Indoor positioning is attracting increased interest from commercial organisations as a method of augmenting and enhancing user experience, in a range of contexts including office buildings, university campuses, museums, airports $[1,2$, $3,4]$ and other public spaces. However, despite the launch of deployment frameworks by major commercial stakeholders, such as Apple's Maps Connect ${ }^{\mathrm{TM}}$, the underlying technologies are still subject to ongoing research, development and refinement. Moreover, indoor positioning is still not widely deployed, nor mature enough to have developed standardized sensor platforms, though much current work focuses on $\mathrm{WiFi}$ fingerprinting techniques $[2,5]$.

In this paper we report on our ongoing work which seeks to develop location-sensitive services to customers of a national chain of wholesale outlets (based in the UK). This project includes software components concerned with positioning, navigation, planning, and user experience. We have experimented with a number of positioning technologies, but in this paper we report specifically on techniques developed using a network of Bluetooth Low Energy (BLE) beacons, deployed on a live commercial site. The full deployment represents 136 beacons over an area of approximately $6000 \mathrm{~m}^{2}$, though we present experimental work using a sub-section of this area (approximately $800 \mathrm{~m}^{2}$ ).

Apple's iBeacon ${ }^{\mathrm{TM}}$ standard was launched in 2013, and since then there has been some interest in developing robust positioning systems on the BLE platform, for example using Received Signal Strength Indicator (RSSI) fingerprinting techniques analogous to those used for $\mathrm{WiFi}$ [1]. However, relatively little work has subsequently addressed Bluetooth based platforms, partially because of the perceived costs of deployment and maintenance. However, unit costs have reduced over time, and manufacturers are increasingly embedding BLE beacons in other electronic devices, making wider scale deployment or networks more practical. In addition, the launch of Google's Eddystone ${ }^{\mathrm{TM}}$ open-standard in 2015 has provided new and richer broadcast formats which have helped develop interest in the wider deployment and embedding of Bluetooth beacon platforms.

We consider that our test site, as a large beacon deployment in a live commercial environment, represents a valuable experimental platform. Most existing work has been conducted on a much smaller scale, and in controlled or constructed environments. Our results will therefore be of interest both to researchers and practitioners using BLE beacons, but also to others working with $\mathrm{WiFi}$ or other radio methods based on asynchronous RSSI measurements from multiple transmitters, in similar environments.

\section{A. Contributions}

The contributions of our work can be summarized as:

- We introduce a framework for user positioning in which locations and pathways are represented by a node-graph. This effectively reduces the positioning inference problem from two dimensions to one, whilst retaining the essential semantic representation of user location (product adjacency).

- We present experimental results obtained using different methods of positioning using asynchronous RSSI measurements from our installed beacon network, specifically: 
exponentially averaged weighted range estimates from beacon-pairs; and a particle-filter method.

- We present results obtained from a large beacon deployment, including experimental analysis of signal characteristics, which will be of interest to researchers and practitioners interested in using beacon platforms in similar contexts.

\section{EXISTING WORK}

There is a growing body of research work concerned with indoor positioning, driven primarily by the desire to augment or personalize the experience of users in a range of contexts [1, $2,3,4]$. Recent work, whilst promising, has highlighted the fact that despite the pervasive use of mobile devices, and technology infrastructures with the potential to provide accurate positioning, reliable positioning is still an open and ongoing research question.

Research is focussed both on specific hardware platforms, and also on suitable processing techniques which are able to robustly infer location from noisy and asynchronous sensor data, often for a user who is moving through a dynamic and unpredictable environment. Much work has attempted to leverage existing hardware infrastructure, such as WiFi. Trilateration techniques, based on known signal strength attenuation (e.g. [6]), are highly sensitive to environmental factors, and much current work instead focusses on refining fingerprint methods, such as those proposed by Zhuang et al. [5] and Hansen et al. [2].

Other recent work has sought to implement pedestrian dead reckoning (PDR) using the sensor array available on commercial mobile platforms; for example, to detect step occurrence and direction [7,8]. There is a growing trend towards the fusion of readings from multiple sensors, in order improve overall accuracy and reliability. For example, Chen. et al. [8] used sparsely deployed Bluetooth beacons to correct drift. Lin et al. [9] used a Kalman Filter to integrate readings from a range of mobile device sensors, including accelerometer, gyroscope, magnetometer and pressure sensor. Li et al. [10] also exploited magnetometer readings, but instead combined them with $\mathrm{WiFi}$ readings in a hybrid fingerprintbased approach.

The tendency towards the use of multiple sensor sources, asynchronously streaming (often noisy) data, has also lead to the adoption of more sophisticated and probabilistic methods of signal and sensor integration. For example, Karlsson et al. [11] used a particle filter to fuse readings from known $\mathrm{WiFi}$ access points, and to further include PDR in the estimation method. Zafari and Papapanagiotou [12] also used a particle filter to improve the tracking accuracy of a beacon-based location system. One significant observation is that despite the large number of techniques and systems that have been proposed, performance is still highly variable. A recent in-situ comparison of 22 systems at the 2014 Microsoft Indoor Localization Competition [15] showed a wide range of positioning accuracy from less than $1 \mathrm{~m}$ to around $10 \mathrm{~m}$. Most of these systems were WiFi based or hybrid systems, but only 3 achieved positioning accuracy of less than $2 \mathrm{~m}$.
Aside from the aforementioned works by Zafari and Papapanagiotou [12] and Chen et al. [8], relatively little work has focussed on Bluetooth beacons as a primary method of positioning. There are likely a number of reasons for this: firstly, Bluetooth beacons are a relatively new platform and not yet widely deployed, whereas $\mathrm{WiFi}$ is fairly ubiquitous in the types of locations of interest. Secondly, some work (for example, Deepesh et al. [13]) has reported problems with signal stability and suggested that this is a limiting factor on accuracy. However, other recent work by Faraghe and Harle $[1,14]$, which reports results obtained with a deployment of 20 beacons over $600 \mathrm{~m}^{2}$ of office space, suggests that better results are obtainable with beacons than with $\mathrm{WiFi}$, using fingerprinting techniques. They also provide some guidelines for deployment, and obtained an accuracy of $2.5 \mathrm{~m}$ with a density of around $30 \mathrm{~m}^{2}$ per beacon. This is comparable with WiFi based systems (e.g. [15]), and does highlight a significant advantage of beacons: existing $\mathrm{WiFi}$ installations have generally not been designed to specifically support positioning. Beacon networks can more easily be configured and adapted to particular environments, and now may also represent a lower installation cost

With this in mind, the work we present here is of particular interest as it reports on the design, deployment and evaluation of a large beacon network in an operational commercial wholesale environment. This environment is typical of other potential use-cases (e.g. retail), and yet there is little existing comparable work.

\section{CONTEXT}

Our project is concerned with enhancing the experience of users whilst shopping at a wholesale store. The store is part of a national chain and users already have access to a mobile app which has been widely adopted by its customer base, across the UK. The objective is to add location-dependent services and data, including navigation information, which can be configured by the user depending on their requirements. We have experimented with a number of positioning systems, but the work we report here is concerned with determining the position in the store, using a network of BLE Beacons and a standard consumer mobile device.

The total deployment is of 136 beacons over a store area of around $6000 \mathrm{~m}^{2}$. However, our experimental data was collected over a subsection of around $800 \mathrm{~m}^{2}$, containing 25 beacons. The store is laid out as a grid, with series of aisles containing typical retail products (food, household items), and connecting aisles running perpendicular. Each aisle comprises a series of tall metal shelving units, as shown in Figure 1. Figure 2 shows an image captured while standing inside one of the aisles.

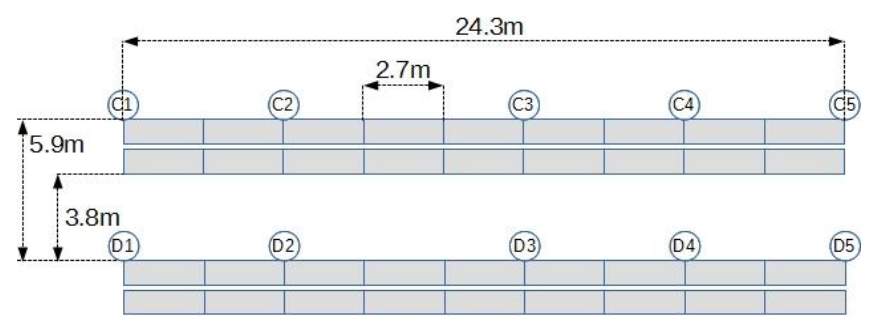

Figure 1: Example aisle dimensions structure. 


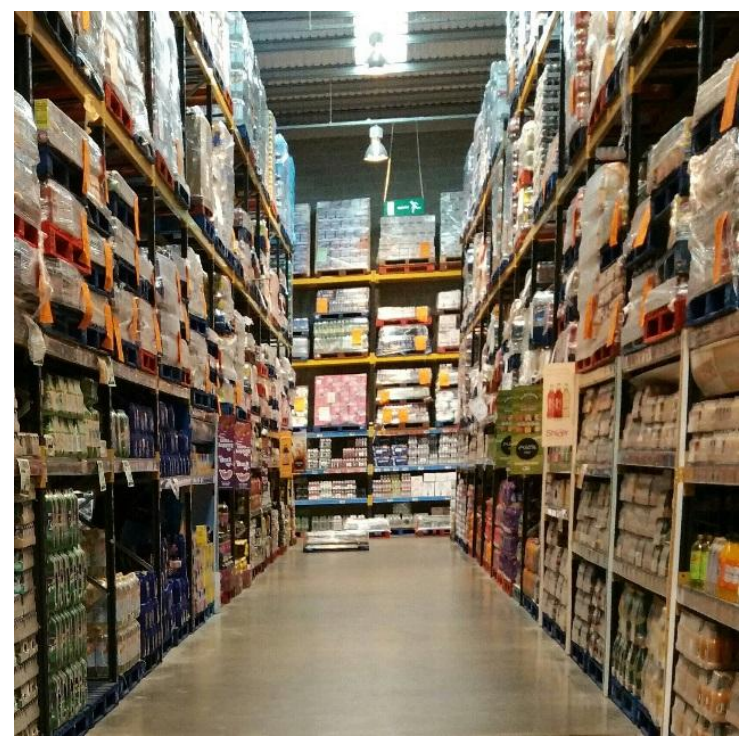

Figure 2: Typical aisle configuration

The objective of our system is to determine which aisle the user is in, and, as accurately as possible, their position along the aisle. This provides enough information to determine which products / product categories are adjacent to the user.

Figure 1 also shows the beacon deployment in the example aisle. Each beacon is depicted as a small circle with a label (e.g. "D1"). The beacon positions are accurately represented: beacons are placed along one side of the aisle, at regular (but not always equal) intervals corresponding to the ends of shelving units. In this case, intervals are either $5.4 \mathrm{~m}$ or $8.1 \mathrm{~m}$, and this is repeated across most of the store. The store comprises a total of 22 separate aisles, ranging from around $24 \mathrm{~m}$ to $60 \mathrm{~m}$ in length, separated by three connecting (perpendicular) aisles of between around $80 \mathrm{~m}$ and $100 \mathrm{~m}$ in length. Beacons are positioned on the shelf units at an approximate height of $2 \mathrm{~m}$. We have used Kontakt.io Smart Beacons for the entire deployment.

\section{THE NODE-GRAPH STORE MAP}

Store aisles are quite long, but relatively narrow (less than $4 \mathrm{~m}$ ): our initial assumption is that signal attenuation between adjacent aisles will be high (due to obstruction/reflection by shelving and product). We therefore anticipate that the signal characteristics will vary more (per unit) distance along the main axis of the aisle than across the width of the aisle. Furthermore, we are primarily interested in determining which products are close to the user: this as fully represented by the displacement of the user along the main axis of the occupied aisle, and the position across the width of the aisle is less important. We have therefore reduced the positioning problem to that of locating the user on an edge of a node graph, which represents a map of the store aisles. A section of map is shown in Figure 3.

The node graph in Figure 3 represents a reduction of the position problem from two dimensions to one: the objective is to locate the user's position with respect to the graph edges (shown in red), or at a node (in yellow). The nodes and edges pass through the centre of the aisle, and we have placed nodes adjacent to each beacon, to more easily capture the geometrical relationship between the beacon and the closest point to it on the aisle centre.

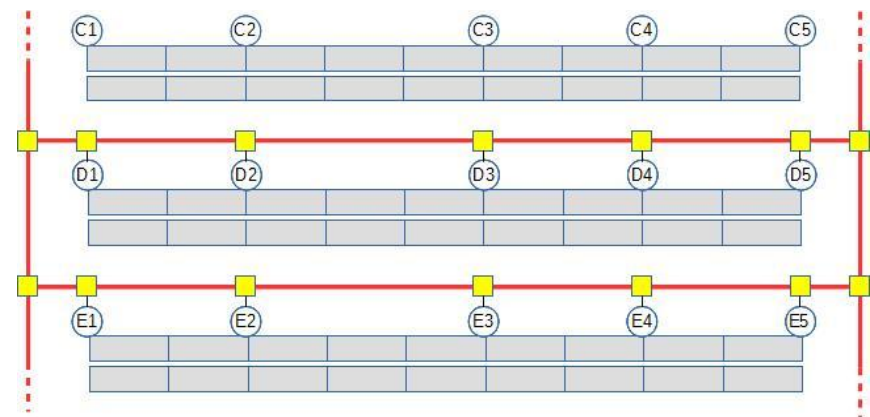

Figure 3: Node graph map representing 2 adjacent aisles. Red lines represent traversable edges, and yellow squares are nodes.

Additional nodes are placed at the centre of junction points. In the example in Figure 3, the nodes on the extreme left and right have additional connections to adjacent aisles (not shown). The intention of this representation is to simplify the positioning problem, whilst retaining the geometrical structure of the aisle and beacon network, spatial relationships between features of the store, and the relevant semantic information about the user's position.

\section{DATa Collection AND PROCESSING}

We have collected sample data from our test area using our own data logging app, developed for the Android operating system. The app logs received BLE readings, and records a beacon identifier, RSSI signal strength, and timing data. In addition, the app allows the user to manually log way points, which are embedded as annotations in the Bluetooth signal data. We collected data by walking continually at a steady pace along a pathway through the centre of three adjacent aisles: those marked D and E in Figure 3, and one below (not shown). We used a Motorola Moto G (Generation 3), held steadily at waist-height to log readings and annotations.

This was done under naturalistic conditions, with shoppers and staff present. We manually recorded a way point at the end of each shelf unit (every $2.7 \mathrm{~m}$ ), and also at each junction intersection point. Between 30 and 50 individual BLE readings were captured from our network between each way point (approximately 1300 total), including readings from beacons in adjacent aisles. We repeated this two further times, to produce a dataset of 3 separate logs, corresponding to the same path.

We developed a separate PC application to load and process the collected data off-line. As the way points are in pre-defined known positions, and assuming a constant walking speed between each, we were able to post-process the data to interpolate a ground-truth position (on the node graph map) for each received BLE reading, using its timing data. We used our app to generate the ground truth positions for the beacon readings, and to conduct our subsequent algorithm development and evaluation. A screen shot is shown in Figure 4. The app was written in $\mathrm{C}++$ using Microsoft Visual Studio 2015, and makes use of the OpenCV image processing library for visualization. 


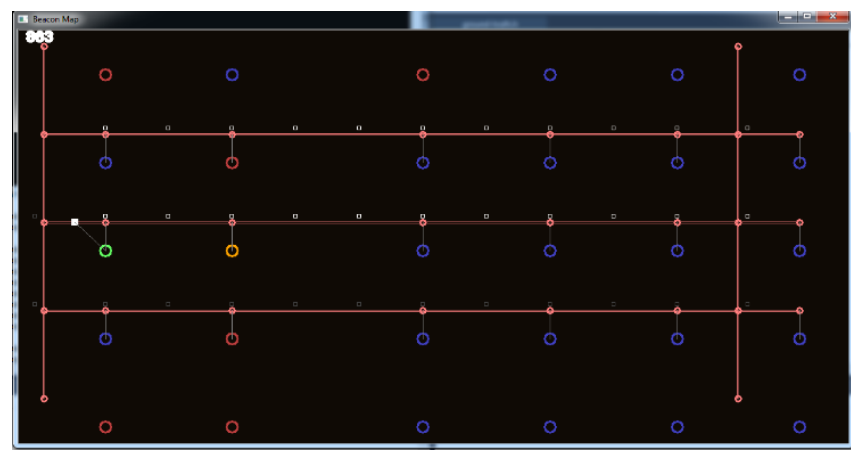

Figure 4: PC-based App for off-line data processing.

\section{Signal CHARACTERISTICS}

The signal attenuation of BLE beacons can be described by the well-known path loss model [16] given by Equation 1:

$$
s_{d}=s_{0}+10 n \log \left(\frac{d}{d_{0}}\right)+N(0, \sigma)
$$

where $s_{d}$ is the measured RSSI (dBm) signal strength at distance $d$ from source, given a value of $s_{0}$ at the reference distance $d_{0}$, and $n$ is the path loss exponent. $\mathrm{N}(0, \sigma)$ is Gaussian noise. However, actual measured values of $S_{d}$ in any particular context are subject to significant variation due to environmental factors including walls, objects, and people.

We use this model to estimate the ranges of observations, and in particular, to develop an observation likelihood function for particle filter-based positioning. We have therefore analysed part of our dataset to determine appropriate model parameters. Note that we take $s_{0}$ to be $-77 \mathrm{dBm}$ at a range of $d_{0}$ $=1 \mathrm{~m}$, in line with manufacturer specifications. We used the first of our 3 BLE reading data sets, with ground truth positions (on the node graph) determined by linearly interpolating the associated timing data between the manually-recorded way points. Given a known perpendicular distance $(1.9 \mathrm{~m})$ between the centre of the aisle and the shelving, and a known relative height of the beacons with respect to the height of the mobile device (approx. 1m), we are able to estimate values of $d$ corresponding to each measured value of $s_{d}$ in Equation 1.

We first split the readings into those taken in the same aisle as their corresponding beacon, and those that were taken in adjacent aisles. This is because we expect those in adjacent aisles to exhibit different characteristics, due to presence of shelving units between the device and the beacon. In each case we binned the data into $1 \mathrm{~m}$ distance intervals, and computed mean values. These are plotted as line graphs of mean $s_{d}$ values against $d$ in Figure 5. Note that whereas the curve generated using measurements taken within the aisle broadly follows the expected lognormal form, those in adjacent aisles do not. The peak in RSSI at around $8 \mathrm{~m}$ is a genuine artefact of the recorded data, and we hypothesize that this is caused by varying densities of shelf contents between the mobile device and beacon sources.

Figure 6 shows the distribution (assuming Gaussian form) of readings taken at distance intervals $2-3 \mathrm{~m}, 4-5 \mathrm{~m}$, and $8-9 \mathrm{~m}$ respectively. As expected, a significant overlap in distribution is observed, and increases with range. This makes accurate measurements from single readings difficult at longer ranges, and further motivates a filtering approach to range estimation.

The path loss exponent $n$ is known to be highly dependent on environmental conditions. Zhao et al. [16] reported measured values of between 1.35 and 1.98 in an office environment. In our case, the graph in Figure 4 is consistent with a value closer to 1.0 , as shown in Figure 7 . For our experimental work, we therefore used a value of $n=1.0$ for estimating ranges of readings made in the same aisle as the source beacon. For those in different aisles, we use a value of 1.3 (following Zhao et al.). Whilst these values are lower than initially anticipated, they correspond well to our data, and are shown to be effective in our results.

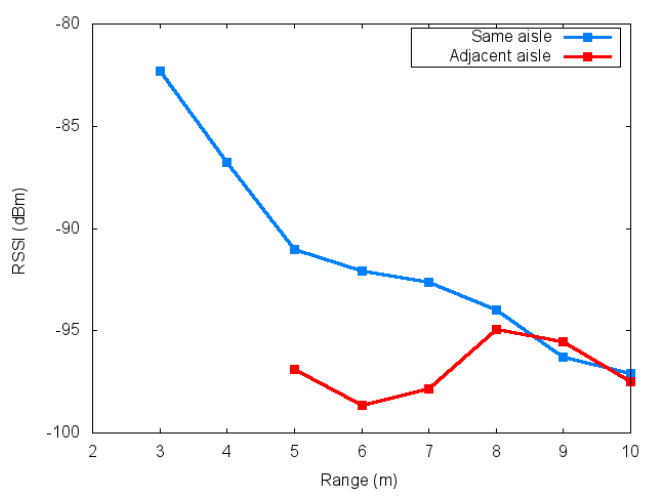

Figure 5: Mean RSSI vs Range for readings taken in the same aisle, and readings taken in adjacent aisles.

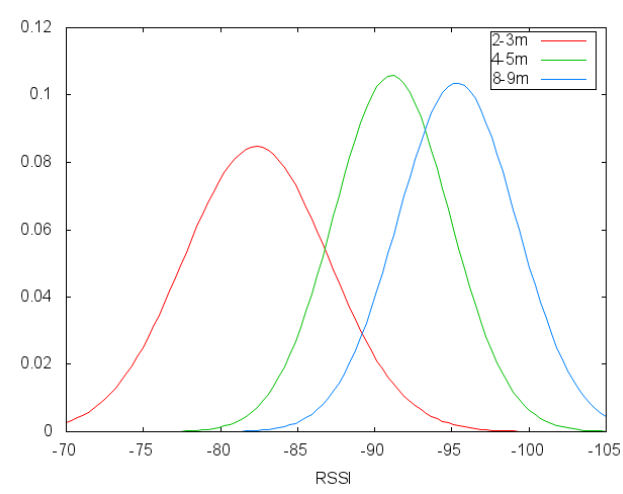

Figure 6: RSSI distributions at varying ranges, recorded in the same aisle as source beacons.

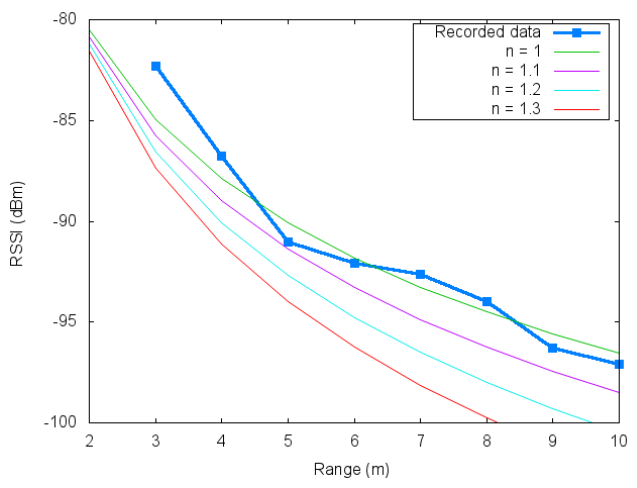

Figure 7: RSSI for the same aisle, and path loss curves for varying $n$. 


\section{Positioning Methods}

We have experimented with three positioning methods, which we have developed using our offline PC application (Figure 4) and datasets. We proceed to describe each in turn, and in the next section present comparative experimental evaluations.

\section{A. Baseline method: Nearest Beacon}

Our baseline method of positioning is to estimate the user's position to be that of the graph node adjacent to the beacon with the current highest recorded RSSI value. There are some complications, however: firstly readings are measured asynchronously, and secondly, individual readings may be differ significantly from those predicted by Equation 1, due to signal noise, obstructions, etc. Given a series $R_{b, t}$ of $k$ readings for a specific beacon $b$ at time $t$, collected over time window $w_{t}$ :

$$
R_{b, t}=\left\{r_{k}, r_{k-1}, r_{k-2}, \ldots, r_{1}\right\}
$$

we have experimented with a two methods for estimating the current signal value for the beacon $S_{b, t}$, given by Equations 3 and 4 respectively:

$$
\begin{aligned}
& S_{b, t}=\alpha r_{k}+(1-\alpha) S_{b, t-1} \\
& S_{b, t}=\max _{i}\left(r_{i} \in R_{b, t}\right)
\end{aligned}
$$

where $\alpha$ is the learning rate parameter for an exponential moving average. The beacon yielding the highest value of $S_{\mathrm{b}, \mathrm{t}}$ at any given time $t$ is taken to be that of the current closest beacon. For our experiments we have used values of $w_{t}$ ranging from 1 to 3 seconds, with little observed difference in performance. We report on results using both methods in Section VIII.

\section{B. Weighted Beacon-Pair Range Estimates}

Our baseline nearest beacon method is inherently limited in resolution, according to the distance between deployed beacons. We wish to incorporate readings from multiple beacons to improve spatial resolution. Trilateration of BLE readings is known to be unreliable [13]; however, the node graph map constrains the user's position, and we use readings from neighbouring beacons to improve the position estimate (by interpolation). From Equation 1, the range of a beacon may be estimated as:

$$
d=d_{0} 10^{\left(\frac{s_{d}-s_{0}}{10 n}\right)}
$$

where $s_{d}$ is the beacon's measured RSSI value (which may be determined using Equations 3 or 4 ), and $s_{0}, d_{0}$, and $n$ are known a priori. In our case, we proceed as follows:

1. We first determine the closest beacon, using either Equation 3 or 4.

2. We then determine the next closest beacon (that is the beacon with the second highest current RSSI value) that lies on the same branch of the node graph (aisle or column). In the majority of cases, this second beacon will be adjacent to the first.

3. Given positions $p_{1}$ and $p_{2}$, the positions of the graph nodes adjacent to the two beacons, and corresponding distance measurements $d_{1}$ and $d_{2}$, calculated using Equation 5, we calculate a target position $p_{t}$ as:

$$
p_{t}=p_{1}+\left(p_{2}-p_{1}\right)\left(\frac{d_{1}}{d_{1}+d_{2}}\right)
$$

Where there are two or more beacons with the same current highest value, this process is repeated and the mean value of $p_{t}$ is calculated. The final estimated current position is calculated using an exponential moving average, with learning rate $\alpha_{\mathrm{p}}$. Again, we present results in Section VIII.

\section{Particle Filter}

Finally, we consider user positioning as a non-linear Bayesian tracking problem. Some previous works (such as that by Zafari and Papapanagiotou [12]) have used particle filter methods for estimating the posterior distribution over a state space, given a time-series of observations. We adopt a comparable approach; however, in our case we constrain the state space to include only positions which lie along node edges of our graph map. This effectively reduces the usual 2 dimensional form $(\mathrm{x}, \mathrm{y})$ to one dimension (distance from a node), and so in principal reduces the number of particles required to effectively model the distribution. A number of popular particle-filtering algorithms exist; in our case we use the Sequential Importance Resampling (SIR) filter (see [17]), which resamples from the distribution after each observation.

The state of an individual particle at time $t$ is defined as $x_{i, t}$ $=\left\{p_{i, t}, v_{i, t}\right\}$ where $p_{i, t}$ is the distance of the particle along a graph edge, and $v_{i, t}$ is its speed of movement towards the node. The particle filter continuously performs a two-step procedure: a prediction step in which a motion model is applied and an update step in which individual weights of particles are updated based on an observation model. The particles are initially randomly distributed throughout the state space, as shown in Figure 8.

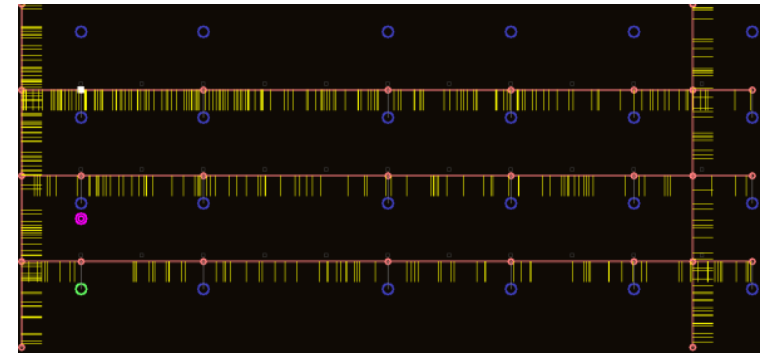

Figure 8: Initialization of the particle filter. Each blue line represents the initial state of an individual particle.

Motion Model. We apply a constant velocity model to predict the next state of each particle. The position of each particle is updated based on its current velocity estimate:

$$
p_{i, t}=v_{i, t} t_{s}
$$

where $t_{s}$ is the time elapsed since the last update. Particles move along their current graph edge until reaching a node. On reaching a node, a new edge is selected randomly, with all edges carrying equal probability. We apply a randomized perturbation to the velocity $v_{i, t}$ of each new sampled particle. Perturbations are uniformly distributed in the range $-V_{d}$ to $+V_{d}$. 
Our experiments include a range of values for $V_{d}$. We also cap $v_{i, t}$ to a maximum value $V_{\max }$.

Observation Model. After receiving an RSSI reading, the weight of each particle is computed. The position of the particle $p_{i, t}$ is known, as is the position of the emitting beacon. Computing the range $d$, and applying Equation 1 gives the corresponding expected RSSI value $s_{d}$. The weight of the particle is then computed as the likelihood of the measured RSSI value, $s_{m}$, at range $d$ :

$$
w_{i, t}=\frac{1}{\sqrt{2 \pi \sigma_{p}^{2}}} e^{-\frac{1}{2}\left(\frac{s_{m}-s_{d}}{\sigma_{p}}\right)^{2}}
$$

where $\sigma_{p}$ is the standard deviation. Figure 5 demonstrates different signal characteristics, depending on whether the user is in the same aisle as the emitting beacon. We therefore parameterize Equations 1 and 8 differently depending on whether particle is in the same branch (aisle or column) of the node graph, or not. We have learned parameters from the data, and present them in Section VIII. We conclude this processing step by normalizing the particle weights:

$$
w_{i, t}=\frac{w_{i, t}}{\sum_{i=1}^{N p} w_{i, t}}
$$

Resampling. After the particle weights have been computed, using Equations 8 and 9, we resample from the posterior distribution. $N_{p}$ new particles are drawn by randomly selecting particles from the existing distribution; the probability of drawing a particle is in proportion to its weight $w_{i, t}$.

Estimating the Position. The user's position at time $t, p_{u}$ is estimated immediately after applying the prediction step as:

$$
p_{u}=\sum_{i=1}^{N p} w_{i, t} p_{i, t}
$$

Example distributions are shown in Figures 9 and 10.

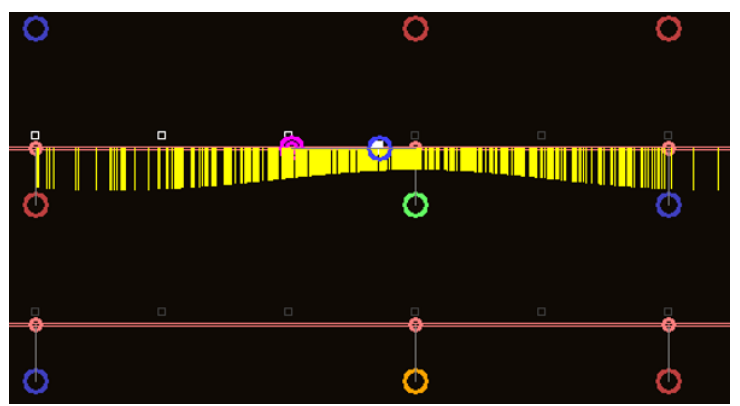

Figure 9: Particles on a Graph Branch.

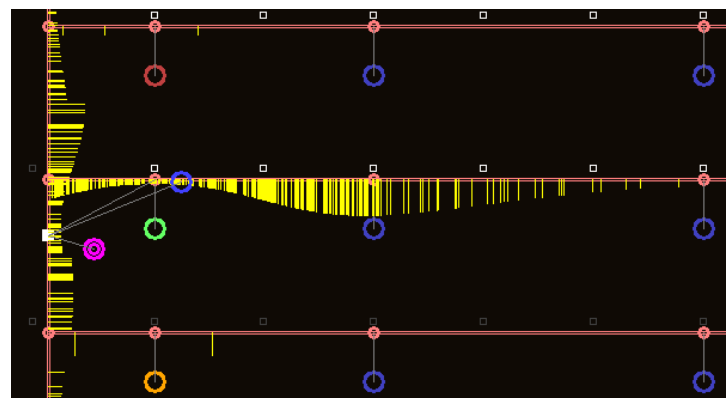

Figure 10: Particles at a Node Junction.

\section{EXPERIMENTS}

We have performed a comparative evaluation of the positioning methods described in section VII. In this section we report our experimental method, parameterization of the algorithms, and results. This is followed by a discussion of the results, conclusion, and directions for further work.

\section{A. Method}

We use our data set, described in Section V, which comprises annotated logs of BLE beacon readings collected while traversing the store. The data was collected while traversing a route of approximately $85 \mathrm{~m}$. The route was repeated three times, and around 1000 - 1300 readings were collected on each repetition (run). Each reading has an associated ground truth position, which is determined from the associated timing data by linearly interpolating between the manual annotations embedded in the log (each annotation corresponds to a known predefined point on the map).

We evaluate each positioning method by using it to estimate the position of the user after each beacon reading, using only the RSSI and timing data. This estimate is compared with the corresponding ground truth position, and the distance between the two is averaged across the entire run (and across all runs). This represents the positioning error.

\section{B. Parameterization}

Each of the three positioning methods requires some degree of parameterization. Using the nearest beacon method (Section VII A), we wish to determine whether the method of averaging the beacon RSSI values (Equation 3) is more effective than taking the maximal value (Equation 4) across a given time window $w_{t}$. We also wish to determine the optimal learning rate $\alpha$ when using Equation 3.

The weighted beacon range method (Section VII A) has a number of parameters. As mentioned, we have pre-determined the values of $s_{0}$ and $n$ in Equation 5 to be $-77 \mathrm{dBm}$ and 1.0 respectively. The only parameter which needs to be determined experimentally is the learning rate $\alpha_{\mathrm{p}}$. We determined this parameter (and the unknown parameters for the nearest beacon method) manually by repeatedly running the algorithms on the first run of data set, and choosing values which minimized the reported error.

The particle filter method has a number of pre-determined parameters. As mentioned, we again took $s_{0}$ in Equation 5 to be $-77 \mathrm{dBm}$ and $n$ to be either 1.0 (same aisle) or 1.3 (different aisle). The number of particles $N_{p}=500$ was used as a baseline for our comparison. The number of remaining parameters, which we wish to estimate experimentally, are: $\sigma_{p}$ in Equation 8 (separately for the case where the particle is in the same aisle as the beacon, and the case when it is not); $V_{d}$, the maximum perturbation applied to the particle velocity on each update, and $V_{\max }$, the maximum velocity of a particle. We automated a parameter search which made repeated error measurements over run 1 from our dataset, and reported the parameter combination returning the lowest mean error. Our results for parameterization are shown in Table 1. 


\begin{tabular}{|l||l|l|}
\hline $\begin{array}{l}\text { Positioning } \\
\text { method }\end{array}$ & Most effective parameters & Mean Error \\
\hline \hline $\begin{array}{l}\text { Nearest } \\
\text { Beacon }\end{array}$ & $\begin{array}{l}\text { Using highest (Eqn. 4) } \\
w_{t}=3.0 \mathrm{~s} \\
\text { Using averaged (Eqn. 3) } \\
w_{t}=3.0 \mathrm{~s}, \alpha=0.25\end{array}$ & $2.15 \mathrm{~m}$ \\
& Averaged $\left(\right.$ Eqn. 3) $w_{t}=3.0 \mathrm{~s}$, & $1.53 \mathrm{~m}$ \\
\hline $\begin{array}{l}\text { Beacon-Pair } \\
\text { Range }\end{array}$ & $\alpha=0.75, \alpha_{\mathrm{p}}=0.5$ & \\
\hline $\begin{array}{l}\text { Particle } \\
\text { Filter }\end{array}$ & $\sigma_{p}($ same aisle) $=4$ & $1.04 \mathrm{~m}$ \\
& $\sigma_{p}($ other aisle $)=6$ & \\
$V_{d}=2.5 \mathrm{~m} / \mathrm{s}$ & \\
& $V_{\max }=4.0 \mathrm{~m} / \mathrm{s}$ & \\
\end{tabular}

Table 1: Parameterization results, using readings from run 1.

It appears from Table 1 that significantly higher accuracy was initially achieved using the particle filter tracking method. This is encouraging, and represents a significantly better positioning accuracy than that reported by most other works [15]. However, there are more operational parameters for the particle filter, and these have been optimized for this data run. In the next section we therefore re-test these algorithms and parameter settings on data from runs 2 and 3.

\section{Results}

Table 2 reports the results achieved using the optimized parameters on runs 1,2 and 3 , and also the totals across all three runs, including mean and standard deviation.

\begin{tabular}{|c|c||c|c|c|c|}
\hline \multirow{2}{*}{ Positioning method } & \multicolumn{3}{|c|}{ Run } & Total \\
\cline { 3 - 5 } & 1 & 2 & 3 & \\
\hline \hline \multirow{2}{*}{$\begin{array}{c}\text { Nearest } \\
\text { Beacon }\end{array}$} & Mean(m) & 2.15 & 2.01 & 1.82 & 2.01 \\
\cline { 2 - 5 } & Std. dev. & 1.49 & 1.47 & 1.35 & 1.44 \\
\hline $\begin{array}{c}\text { Beacon- } \\
\text { Pair } \\
\text { Range }\end{array}$ & Mean(m) & 1.53 & 1.78 & 2.03 & 1.76 \\
\cline { 2 - 5 } & Std. dev. & 1.45 & 1.62 & 1.72 & 1.59 \\
\hline $\begin{array}{c}\text { Particle } \\
\text { Filter }\end{array}$ & Mean(m) & 1.04 & 1.07 & 1.44 & 1.16 \\
\cline { 2 - 5 } & Std. dev. & 0.97 & 1.24 & 1.31 & 1.18 \\
\hline
\end{tabular}

Table 2: Results for all data runs.

Significance of the results. A two sampled t-test of the totals for the particle filter and beacon-pair range methods yields a t-value of -18.3 and $99 \%$ confidence that the mean error value achieved with the particle filter is $0.5982 \pm 0.086$ lower (assuming unequal variances). A similar comparison the of the particle filter and nearest beacon method yield 99\% confidence that the mean error value of the particle filter method is $0.8403 \pm 0.08$ lower. This analysis demonstrates that the particle filter method is significantly superior to the other two evaluated methods.

Number of Particles. Having established the superiority of the particle filtering method, we have further investigated the effects of varying the numbers of particles. We Repeated the evaluation using $N_{p}=250$ and $N_{p}=1000$. In each case, we reoptimized the other parameters also. Results are shown in Table 3.

\begin{tabular}{|l|c||c|c|c|c|}
\hline \multicolumn{2}{|c||}{\begin{tabular}{c} 
Number of particles \\
\multicolumn{2}{|c||}{$N_{p}$}
\end{tabular}} & \multicolumn{3}{c|}{ Run } & Total \\
\cline { 2 - 5 } & Mean(m) & 1.09 & 1.11 & 1.90 & 1.33 \\
\hline \hline $\begin{array}{l}250 \\
\sigma_{p}=4,6 \\
V_{d}=3.0 \mathrm{~m} / \mathrm{s} \\
V_{\max }=6.0 \mathrm{~m} / \mathrm{s}\end{array}$ & Std. dev. & 1.03 & 1.26 & 1.68 & 1.32 \\
\cline { 2 - 5 } & Mean(m) & 1.04 & 1.07 & 1.44 & 1.16 \\
\hline $\begin{array}{l}500 \\
\sigma_{p}=4,6 \\
V_{d}=2.5 \mathrm{~m} / \mathrm{s} \\
V_{\max }=4.0 \mathrm{~m} / \mathrm{s}\end{array}$ & Std. dev. & 0.97 & 1.24 & 1.31 & 1.18 \\
\cline { 2 - 5 } $\begin{array}{l}1000 \\
\sigma_{p}=4,5 \\
V_{d}=2.5 \mathrm{~m} / \mathrm{s} \\
V_{\max }=4.0 \mathrm{~m} / \mathrm{s}\end{array}$ & Std. dev. & 0.98 & 1.17 & 1.36 & 1.17 \\
\cline { 2 - 5 } & Mean(m) & 1.05 & 1.02 & 1.43 & 1.15 \\
\hline
\end{tabular}

Table 3: Results for particle filter with varying $N_{p}$.

It can be seen from Table 3 that the performance using $N_{p}$ $=250$ particles is worse than that recorded using $N_{p}=500$ particles (the mean of total errors is $17 \mathrm{~cm}$ higher). Using $N_{p}=$ 1000 particles produces only a slight improvement in performance over $N_{p}=500$.

\section{CONCLUSIONS AND FURTHER WORK}

We have presented our work on indoor positioning of shoppers, using standard mobile hardware and an array of BLE beacons deployed in a wholesale store. As a case study, this is of interest to researchers and practitioners as it represents a commonly discussed use-case for indoor positioning (shopping), yet relatively little work has used live data from such environments using a BLE platform.

We have proposed a mapping scheme which represents the environment as a node graph traversed by the user, and leveraged this representation in our positioning algorithms to constrain our search space. We have further evaluated three proposed positioning methods: nearest beacon (baseline), averaged beacon-pair ranging, and a particle filter based tracking method. We have presented results from three separate data runs in the same section of the store, over a pathway of length $85 \mathrm{~m}$. Using inferred ground truth positions for collected beacon readings, we have made average error estimates for each method, and found that the particle filter method outperforms the others significantly, as indicated in Table 2 . We have further investigated the effects of varying the numbers of particles, and found that increasing from 500 to 1000 particles has a relatively small improvement on performance, whilst reducing to 250 particles causes a more noticable reduction in performance.

We wish to further develop this work to support our wider project aims (providing location-specific user experience to shoppers), and conduct further evaluations which include the entire store area. Furthermore, we wish to develop the 
promising particle filtering method to include data from other on-board sensors (such as accelerometer) to further refine and improve the accuracy of the positioning system. We also wish to further evaluate the performance and dynamic scalability of the particle filter system, on consumer mobile devices.

\section{ACKNOWLEDGMENTS}

The work presented in this has been supported by Innovate UK, and been conducted in collaboration with our project partners, RNF Digital Innovation Ltd, and Bestway Cash and Carry Ltd.

\section{REFERENCES}

[1] R. Faragher and R. Harle, "Location fingerprinting with bluetooth low energy beacons", IEEE Journal on Selected Areas in Communication, 33(11), pp. 2418-2428, 2015.

[2] R. Hansen, B. Thomsen, L. L. Thomsen, and F. S. Adamsen, "SmartCampusAAU - an open platform enabling indoor positioning and navigation," in Proceedings of 14th IEEE International Conference on Mobile Data Management, vol. 2, pp. 33-38, 2013.

[3] Z. He, B. Cui, W. Zhou and S. Yokoi, "A proposal of interaction system between visitor and collection in museum hall by iBeacon," In Proceedings of 10th International Conference on Computer Science \& Education (ICCSE), Cambridge, pp. 427-430, 2015.

[4] S. He, S. H. G. Chan, L. Yu and N. Liu, "Fusing noisy fingerprints with distance bounds for indoor localization," In Proceedings of IEEE Conference on Computer Communications (INFOCOM), Kowloon, pp. 2506-2514, 2015.

[5] Y. Zhuang, J. Yang, Y. Li L. Qi and N. El-Sheimy, "Smartphone-Based Indoor Localization with Bluetooth Low Energy Beacons", Sensors 16(5), 596, 2016.

[6] Z. Yang and Y. Liu, "Quality of Trilateration: Confidence-Based Iterative Localization," in IEEE Transactions on Parallel and Distributed Systems, vol. 21, no. 5, pp. 631-640, May 2010.

[7] Z. Xiao, H. Wen, A. Markham, N. Trigoni, "Robust Pedestrian Dead Reckoning (R-PDR) for Arbitrary Mobile Device Placement", In Proceedings of the IEEE International Conference on Indoor Positioning and Indoor Navigation (IPIN), 27-30 October 2014.
[8] Z. Chen, Q. Zhu, H. Jiang and Y. C. Soh, "Indoor localization using smartphone sensors and iBeacons," in Proceedings of 10th IEEE Conference on Industrial Electronics and Applications (ICIEA), Auckland, pp. 1723-1728, 2015.

[9] T. Lin, L. Li and G. Lachapelle, "Multiple sensors integration for pedestrian indoor navigation," In Proceedings of the International Conference on Indoor Positioning and Indoor Navigation (IPIN), 2015.

[10] Y. Li, Z. He, J. Nielsen and G. Lachapelle, "Using Wi-Fi/magnetometers for indoor location and personal navigation," In Proceedings of the International Conference on Indoor Positioning and Indoor Navigation (IPIN), 2015.

[11] F. Karlsson, M. Karlsson, B. Bernhardsson, F. Tufvesson and M. Persson, "Sensor fused indoor positioning using dual band WiFi signal measurements," In Proceedings of European Control Conference (ECC), Linz, pp. 1669-1672, 2015.

[12] F. Zafari and I. Papapanagiotou, "Enhancing iBeacon Based MicroLocation with Particle Filtering," in Proceedings of IEEE Global Communications Conference (GLOBECOM), San Diego, CA, 2015.

[13] P. C. Deepesh, Rashmita Rath, Akshay Tiwary, Vikram N. Rao, and N. Kanakalata. 2016. "Experiences with using iBeacons for Indoor Positioning", In Proceedings of the 9th India Software Engineering Conference (ISEC '16). 2016.

[14] R. Faragher, R. Harle, "An Analysis of the Accuracy of Bluetooth Low Energy for Indoor Positioning Applications", Proceedings of the 27th International Technical Meeting of the Satellite Division of the Institute of Navigation, Tampa, Florida, September 2014.

[15] D. Lymberopoulos, J. Liu, X. Yang, R. R. Choudhury, V. Handziski, and S. Sen, "A realistic evaluation and comparison of indoor location technologies: experiences and lessons learned", In Proceedings of the 14th International Conference on Information Processing in Sensor Networks (IPSN '15). ACM, New York, NY, USA, 178-189. 2015.

[16] X. Zhao, Z. Xiao, A. Markham, N. Trigoni and Y. Ren, "Does BTLE measure up against WiFi? A comparison of indoor location performance," European Wireless 2014; 20th European Wireless Conference; Proceedings of, Barcelona, Spain, 2014, pp. 1-6.

[17] M. S. Arulampalam, S. Maskell, N. Gordon, and T. Clapp, "A tutorial on particle filters for online nonlinear/non-gaussian bayesian tracking," Signal Processing, IEEE Transactions on, vol. 50, no. 2, pp. 174-188, 2002. 